\title{
A EFICÁCIA DA TCC PARA O \\ ENFRENTAMENTO DA HOSPITALIZAÇÃO \\ EM CRIANÇAS COM CÂNCER: UMA \\ REVISÃO SISTEMÁTICA
}

DOI: $10.22289 / 2446-922 X . V 2 N 2 A 4$

Licinere Silva Ribeiro ${ }^{1}$

Résia Silva de Morais

\section{RESUMO}

O estudo tem como objetivo assimilar os métodos, e as técnicas de intervenções eficazes, e a partir disso, realizar uma comparação dos resultados pertinentes ao tratamento da criança hospitalizada, diagnosticada com câncer, por meio da Terapia Cognitivo-comportamental, a partir de uma revisão sistemática da literatura. O método empregado para o apuramento dos artigos se deram de natureza quali-quantitativo descritivo. A busca dos materiais foi através de publicações eletrônicas em cinco bases de dados a saber: SciELO, PubMed, PsycINFO, PePsic e Springerlink. Ficando inclusos textos completos publicados no período entre 2001 a 2016, em idiomas de Português e Inglês. Observou-se que a TCC pode contribuir para o tratamento de crianças hospitalizadas com câncer, por meio de diferentes técnicas, como entrevista gravada, reestruturação cognitiva, relaxamento e atividades lúdicas. Concluiu-se que as intervenções da TCC se apresentam eficazes para o tratamento de crianças hospitalizadas com câncer, podendo estas, contribuírem para se obter resultados significativos diante da minimização do impacto psicológico do câncer infantil.

Palavras-chave: Câncer, Enfrentamento, Hospitalização, Terapia CognitivoComportamental, Crianças.

\section{ABSTRACT}

The study aims to assimilate the methods and techniques of effective interventions, from there, to make a comparison of the relevant results for the treatment of hospitalized children diagnosed with cancer through cognitive-behavioral therapy, from a systematic review of the literature. The method used to calculate the articles are given descriptive qualitative and quatitativo nature. The search of the materials was through electronic publications in five databases namely: SciELO, PubMed, PsycINFO, PEPSIC and SpringerLink. Getting full texts published between 2001-2016 included in Portuguese and English. It was observed that CBT

\footnotetext{
${ }^{1}$ Endereço eletrônico de contato: licinere@hotmail.com
} 
can contribute to the treatment of children hospitalized with cancer, through different techniques, such as recorded interviews, cognitive restructuring, relaxation and leisure activities. It was concluded that the interventions of CBT have proven effective for the treatment of hospitalized children with cancer and can help to achieve significant results in minimizing the psychological impact of childhood cancer.

Kewords: Cancer, Coping, Hospitalization, Cognitive behavioral therapy, Children.

\section{INTRODUÇÃO}

O câncer e a hospitalização da criança apresentam uma série de sensações desagradáveis no decorrer do tratamento, além disso, podem provocar alterações no desenvolvimento psicológico e emocional, entretanto é necessário que haja todo um preparo para que a mesma não se sinta tão afetada diante da doença.

$\mathrm{Na}$ atualidade a literatura tem apresentado um conceito para interpretar o enfrentamento à doença (coping) e o mesmo foi levado para a oncologia, mais especificamente relacionada ao modo como os pais enfrentam o câncer ${ }^{(1)}$.

Crianças com câncer são expostas a hospitalizações prolongadas e frequentes, e poderão sofrer rupturas na sua condição de vida normal, a criança se depara abruptamente, em um hospital, às vezes, indefesa e expectadora diante da situação que a envolve, passando a conviver com uma série de procedimentos terapêuticos, muitas vezes invasivos e dolorosos. Sendo assim, é necessário que haja técnicas de intervenções psicológicas para um melhor enfrentamento da doença ${ }^{(2)}$.

O câncer infantil tem apresentado a segunda maior causa de morte no Brasil, com cerca de 9000 casos novos, diferentemente dos adultos, o câncer na infância afeta geralmente as células do sistema sanguíneo e dos tecidos de sustentação, sendo que os atuais métodos de tratamento são mais eficazes e suas chances de cura são muito maiores do que nos adultos. Verifica-se que em torno de $70 \%$ das crianças adoecidas pelo cancro podem ficar saudáveis, se avaliadas precocemente e cuidadas em centros especializados ${ }^{(3)}$.

A chance de cura, não alivia a necessidade de que a criança seja submetida a procedimentos invasivos e dolorosos, como é o caso da quimioterapia, um dos recursos frequentemente utilizados contra o câncer na infância(4). A quimioterapia possui efeitos colaterais como o mal-estar geral, febre, vômitos, diarreia, úlceras na boca, queda do cabelo, 
imunodepressão, entre outros, sugerem que a mesma se constitui, de fato, uma segunda doença para a criança e sua família(5).

Através dessa experiência particular da criança, bem como da maneira que se deu o enfrentamento dessa situação, que os sentimentos afloram e permeiam essa vivência. Segundo Moraes e Assis ${ }^{(6)}$ o câncer, coloca à criança em sofrimento e estabelece um certo grau de desespero que altera sua vida, e gera expressões de pena e de pesar decorrentes do medo. A partir do momento que a criança adoece, sua vida passa por uma rápida e intensa transformação, independentemente de sua idade e de sua capacidade de compreensão cognitiva da realidade que a rodeia ${ }^{(7)}$.

O tratamento do câncer infantil em geral ocorre com a hospitalização da criança, tanto ela quanto a família passam por uma mudança abrupta. A partir do momento que o diagnóstico é mencionado, muitas das vezes a criança é afastada do lar por um período indeterminado. As maneiras de enfrentamento da doença e do tratamento variam de acordo com o modo como cada família lida com tais situações adversas ${ }^{(9)}$.

Logo após o diagnóstico, muitas crianças se deparam com a dúvida em relação ao futuro, pois a sensação de perda é uma questão com a qual a criança enfrentará, necessitando do apoio da família e de amigos. Além disso, terá que se submeter a normas e tratamentos impostos pela equipe cuidadora, perdendo sua privacidade e liberdade, ao mesmo tempo, um novo significado ao mundo e a vida(10).

Diante de tudo isso, a Oncologia Pediátrica é considerada como a área médica que estuda o câncer infantil, e faz parte da Psicologia da Saúde, pois estuda a atuação de fatores psicológicos sobre o crescimento e a causa do câncer infantil ${ }^{(11)}$. Neste caso esta área da psicologia pode ser intitulada como Psico-oncologia.

A psico-oncologia ao propiciar condições de qualidade de vida ao paciente com câncer e sua família, auxilia no processo de conhecimento e reconhecimento de si mesmo diante da doença, aprendendo sobre os sintomas e as possibilidades terapêuticas ${ }^{(12)}$.

Através da formação em Psico-oncologia é possível notar o cuidado aos pacientes com câncer que, e a ligação da Psicologia e a Oncologia, resultando assim um maior conhecimento a respeito destas duas áreas de trabalho. Este conhecimento possibilita a ampliação destas duas especialidades dentro de uma perspectiva de trabalho que vise à valorização dos aspectos emocionais do paciente oncológico e a inclusão destes fatores no seu tratamento ${ }^{(13)}$. 
$\mathrm{Na}$ psico-oncologia destaca-se a intervenção psicoterápica breve, que requer entendimento e experiência, sendo assim, poderá amenizar a ansiedade e outros sintomas clínicos, na qual o psicoterapeuta poderá se apresentar no papel de orientador, educador e protetor permanente. O discurso deverá ser o interpretativo, escutando profundamente as representações, interpretações e fantasias do paciente diante do câncer e do tratamento, dessa maneira, compreende-se que há um grande desafio na prática da psico-oncologia ${ }^{(14)}$.

$\mathrm{Na}$ área da saúde, portanto, se observa a necessidade de adaptação de técnicas em psicologia clínica no contexto hospitalar, inclusive na atuação clínica individual. No contexto hospitalar, as intervenções em grupos operativos têm trazido grandes resultados, para a prevenção e promoção de saúde junto ao psicólogo hospitalar(15).

De tal modo, a Psico-oncologia configura um desafio para os profissionais da Psicologia, diante disso, cabe aos acadêmicos e, aos profissionais da área inteirarem-se de métodos eficazes de intervenção para o enfrentamento da hospitalização em crianças com câncer, de modo a atuar com aptidão. Eis, então, a relevância científica deste trabalho, que se preocupa em apreciar métodos, diferentes técnicas e resultados referentes ao tratamento de crianças hospitalizadas com câncer, através da Terapia Cognitiva-Comportamental (TCC).

A TCC, foi fundada por Aaron Beck na década de 60, e compõe-se de uma abordagem diretiva, objetiva, de tempo limitado e constituído dentro do método científico ${ }^{(16)}$.

Para esses autores, a forma como um sujeito analisa situações específicas, influência seus sentimentos, motivações e ações dessa maneira, o foco do modelo cognitivo está na interação entre pensamentos, sentimentos e comportamentos. A TCC pode oferecer contribuições para o enfrentamento da hospitalização de crianças com câncer, por meio de técnicas como o brincar que se insere como uma tentativa de transformar o ambiente hospitalar, proporcionando assim melhores condições psicológicas ${ }^{(17)}$.

O brincar pode ser concebido como próprio da saúde infantil e definido como objetivo da psicoterapia desenvolver ou recuperar essa capacidade na criança, por ser um canal de resolução de maldades, bondades, medos, superação de desafios, crescimento, constituindo, portanto, um aspecto essencial no processo de desenvolvimento saudável do indivíduo ${ }^{(18)}$.

Sem habilidades de autorregulação e de autocontrole, as crianças podem ser incapazes de ir até o fim das intervenções, a partir disso as estratégias de relaxamento reduzem os sintomas, para que outras técnicas sejam mais eficazes, melhorando assim o funcionamento emocional das crianças ${ }^{(19)}$. 
Os terapeutas procuram identificar as áreas que necessitam de intervenções e construir as habilidades do paciente conforme as características do mesmo. A influência da modelagem do desenvolvimento de novos comportamentos é estável, as crianças aprendem a partir da observação de seus contextos, aumentando assim o sucesso das técnicas(20).

Comportamentos repetitivos, por exemplo, como tiques ou hábitos nervosos, levam a problemas sociais e físico, a partir disso a criança fica cada vez mais atento a aparência, e ao praticar o enfrentamento em terapia a mesma passa a se acostumar e aceitar o tratamento. Métodos de autoinstrução representam a primeira tentativa de intervenções da Terapia Cognitivo-Comportamental para lidar com pensamentos disfuncionais e perturbadores. A reestruturação cognitiva mostra a criança que, se é possível mudar seus pensamentos, também é possível mudar as sensações(21).

As técnicas comportamentais minimizam a frequência e gravidade de comportamentos indesejados, ao mesmo tempo em que aumentam a frequência de comportamentos desejados. Somado a isso, mudanças nas atitudes, nas emoções e na cognição resultam em mudanças de comportamento(22).

As técnicas mais utilizadas no tratamento do câncer parte dessas técnicas de intervenção, a aspecto lúdico, por exemplo, em que o brinquedo tem seu papel de diversão estendida à expectativa de construção de sentimentos e conhecimentos de novos comportamentos $^{(23)}$.

Os procedimentos de coping das crianças são mais específicos e estão de acordo com a evolução cognitiva, social e emocional. As estratégias mais frequentes, utilizadas são o domínio do perigo, a busca de aceitação, a solução de problema e a distração, enquanto as menos frequentes envolvem agressão, autodestruição e afastamento. É interessante destacar um aspecto do coping infantil em relação ao comportamento de esquivar-se de estratégias focalizadas na emoção, que podem funcionar de forma adaptativa quando a criança não pode mudar a situação ou quando evoca muita emoção, sendo assim uma tentativa de manter o controle sobre a situação(24).

Para tanto, este estudo tem como objetivo, assimilar os métodos e as técnicas de intervenção eficazes, e a partir disso, realizar uma comparação dos resultados pertinentes ao tratamento da criança hospitalizada, diagnosticada com câncer, por meio da Terapia Cognitivo-comportamental, a partir de uma revisão sistemática da literatura. 


\section{METODOLOGIA}

O presente estudo é definido como uma revisão sistemática quali-quantitativa descritiva permitindo a interpretação e análise das circunstâncias do problema estudado(25). Caracteriza-se como uma Revisão Sistemática da Literatura, assim como outros tipos de estudo de revisão, é uma forma de pesquisa que utiliza como fonte de dados à literatura sobre determinado tema. Esse tipo de investigação proporciona um resumo das evidências relacionadas a uma estratégia de intervenção específica, mediante a aplicação de métodos explícitos e sistematizados de busca, apreciação crítica e síntese da informação selecionada(26,27)

A busca dos materiais foi através de publicações eletrônicas em cinco bases de dados a saber: SciELO, PubMed, PsycINFO, PePsic e Springerlink. Ficando inclusos textos completos, em idiomas de Português e Inglês. Os descritores de busca foram: 'estratégias de enfrentamento and câncer infantil', 'coping and child and câncer'. O período de publicação dos estudos consultados para confeccionar a base teórica de todo o trabalho, foi de 1976 a 2016. Mas, para realizar a análise sistemática o período de publicação dos estudos foi de 2001 a 2016. Essa seleção se justifica por serem bases reconhecidas e por serem bastante utilizadas em revisões sistemáticas da literatura nas áreas de saúde e Psicologia.

Inicialmente, foram analisados os títulos dos artigos com o intuito de eliminar referências repetidas, em cada busca. Em seguida, os resumos anexados foram selecionados e uma leitura prévia dos mesmos determinou a seleção do material necessário para as revisões.

Para as análises seguiu os seguintes critérios de inclusão: I) todas as palavras do descritor presente no corpo do texto; II) idioma de publicação - artigos publicados na integra em língua inglesa e portuguesa; III) formato - somente as referências publicadas como pesquisa de campo foram selecionadas. Consequentemente, foram descartadas as referências publicadas como livro, capítulo de livro, dissertação, tese, resenha, resumo ou carta ao editor; IV) referências repetidas em outras bases foram selecionadas apenas em uma base, sendo descartadas nas outras e V) referências que estavam relacionadas ao tema, tendo como critério norteador do estudo o enfoque sobre a Intervenção psicológica diante do enfrentamento da hospitalização de crianças com câncer, por meio da terapia cognitivocomportamental, além de estudos que empregam esses instrumentos na área hospitalar em geral. Os dados estatísticos foram analisados com auxílio do programa SPSS.

Rev. Psicol Saúde e Debate. Jan., 2017:2(2):58-75. 
Utilizando os critérios para inclusão das referências, os artigos selecionados foram recuperados na íntegra, formando o corpus que delimitou o material de análise. Posteriormente, foi feito um tabelamento do material e os achados dessa organização foram analisados seguindo a identificação de sete dimensões de análise, a saber: (1) Autores/Ano; (2) Amostra; (3) Intervenção; (4) Objetivo; (5) Técnicas; (6) Resultados e (7) Conclusões.

\section{RESULTADOS}

\subsection{Apreciação quantitativa das referências}

Após o uso dos critérios de seleção das referências, a base de dados SciELO possibilitou identificar um total de 8 artigos relacionados com os descritores dos quais 6 foram selecionados. Na PubMed, a busca resultou em 182 referências de estudos publicados em periódicos dos quais 3 foram selecionados. Para a base de dados Psyclnfo foram encontrados 6 artigos, na qual não foi incluída nenhuma referência. Na base de dados Springerlink, não foi encontrada nenhuma referência. A base de dados Pepsic possibilitou encontrar um total de 5 artigos, entre eles apenas 1 referência foi aproveitada. Os dados da revisão foram tabelados, resultando num total de 10 artigos.

A tabela 1 apresenta o processo de seleção dos artigos da revisão, e o total cujas referências foram avaliadas qualitativamente seguindo algumas dimensões de análise. 
Tabela 1 - Percurso de seleção dos artigos da revisão da hospitalização da criança com câncer e as técnicas Cognitivo-Comportamental.

\begin{tabular}{|c|c|c|c|c|c|c|}
\hline $\begin{array}{l}\text { Base de } \\
\text { Dados }\end{array}$ & Descritores & Limites & $\begin{array}{l}\text { № referências } \\
\text { encontradas }\end{array}$ & $\begin{array}{l}\text { Após } \\
\text { Critérios de } \\
\text { seleção } \\
\text { (5 Critérios) }\end{array}$ & $\begin{array}{l}\text { №. de } \\
\text { referências } \\
\text { selecionadas }\end{array}$ & $\begin{array}{l}\text { Dimensões de } \\
\text { análise } \\
\text { (7 Dimensões } \\
\text { Apêndices) }\end{array}$ \\
\hline Scielo (1) & $\begin{array}{l}\text { "Estratégias de } \\
\text { enfrentamento } \\
\text { and câncer infantil" }\end{array}$ & $\begin{array}{l}\text { Com a frase exata; } \\
\text { - Referências } \\
\text { publicadas entre } \\
2001 \text { e } 2016\end{array}$ & 8 & - & 6 & - \\
\hline PubMed (2) & $\begin{array}{l}\text { "Coping and chid } \\
\text { and cancer" }\end{array}$ & - Todos os Índices. & 182 & - & 3 & - \\
\hline Pepsic (3) & $\begin{array}{l}\text { "Estrategias de } \\
\text { enfrentamento } \\
\text { and cancer infantil" }\end{array}$ & $\begin{array}{l}\text { - Com a frase exata; } \\
\text {-Referências } \\
\text { publicadas entre } \\
2001 \text { e } 2016\end{array}$ & 5 & - & 1 & - \\
\hline Psyclnfo (4) & $\begin{array}{l}\text { "Coping and chid } \\
\text { and cancer" }\end{array}$ & - Todos os Índices. & 6 & - & 0 & - \\
\hline Springerlink(5) & $\begin{array}{l}\text { "Coping and chid } \\
\text { and } \\
\text { cancer" }\end{array}$ & - Todos os İ́ndices. & 0 & - & 0 & - \\
\hline Total & & - & 201 & _ & 10 & - \\
\hline
\end{tabular}

Rev. Psicol Saúde e Debate. Jan., 2017:2(2):58-75. 


\section{DISCUSSÃo}

\subsection{Apreciação qualitativa das referências}

A avaliação da primeira dimensão (Autores/Ano de publicação) foi escolhida pelo fato de que, durante o processo de seleção, observaram-se referências com autores repetidos. Dos 10 artigos, seis referências ${ }^{(28,29,30,31,32,33)}$ eram dos mesmos autores, apesar da ordem de importância dos articulistas ser variável. As quatro referências restantes ${ }^{(34,35,36,37)}$ são a partir do ano de 2006, sendo que os autores e os assuntos diferenciam-se.

Já a segunda dimensão (Amostra) revela que os participantes eram crianças e adolescentes dos 6 aos 12 anos. Todos os autores exceto Beltrão et al(34) utilizaram de técnicas de observação e entrevista gravada, para conhecer a percepção materna frente ao câncer infantil e as estratégias de enfrentamento. Outros autores como Motta e Enumo ${ }^{(29)}$ e Motta e Enumo ${ }^{(30)}$ analisaram a importância do brincar no hospital, e avaliaram as estratégias de enfrentamento da hospitalização, a partir de relatos de crianças, meninas e meninos, entre 6 a 12 anos, inscritas no sistema público para o tratamento do câncer.

A terceira dimensão de análise (Intervenção) foi compreendida por técnicas da terapia cognitivo-comportamental, tais como reestruturação cognitiva, relaxamento; através de intervenções lúdicas nos hospitais com objetivo de incentivar a interação ágil e sensível das crianças hospitalizadas. Motta e Enumo ${ }^{(29)}$ e Motta e Enumo ${ }^{(30)}$ por exemplo, utilizaram estratégias de intervenção na hospitalização das crianças. Os mesmos autores manusearam 41 pranchas ilustradas, divididas nos conjuntos: a: enfrentamento da hospitalização, e b: brincar no hospital. Tendo como proposta, avaliação das estratégias de enfrentamento da hospitalização em crianças com câncer, buscando contribuir para o atendimento hospitalar e o direcionamento de intervenções, que tornem o apoio social mais adequado às instituições Hospitalares.

Já Motta e Enumo ${ }^{(33)}$ avaliaram os efeitos comportamentais de um programa de intervenção psicológica lúdica em 12 crianças com câncer, de 7 a 12 anos, internadas em hospital público infantil. Deste modo, a inclusão dessas crianças, foi feito segundo a ordem de chegada à enfermaria, de modo que a primeira criança fosse dirigida ao G1, a segunda ao G2, e assim, sucessivamente. O programa relatava a elevação de estratégias de enfrentamento, favoráveis à hospitalização, minimizando assim, estratégias possíveis de colocar a adaptação e o desenvolvimento da criança em risco. Em Morais e Enumo(32) as 
mães de crianças entre 6 e 12 anos responderam individualmente, um Instrumento de Avaliação Informatizada do Enfrentamento da Hospitalização que permite identificar a eficácia das estratégias de enfrentamento. Hildenbrand et al. ${ }^{(36)}$ desenvolveu intervenções com a família, através de técnicas de enfrentamento dos fatores de stress, relacionados ao câncer, e de acordo com o levantamento das estratégias realizadas com as crianças entre 6 a 12 anos, confirmou o apoio emocional e social das mesmas. Já Beltrão et al. ${ }^{\left({ }^{(3)}\right)}$ utilizou técnicas de observação e entrevista gravada, através de um roteiro sistemático norteando três questões, tais como: "como você se sentiu quando descobriu que seu filho tinha câncer? ", "O que significou para você participar da admissão social? ", "O que tem ajudado você a enfrentar essa experiência?". Já no caso de Hoster et al. ${ }^{(28)}$ as crianças entre 6 a 12 anos foram avaliadas pelo Instrumento Informatizado de Avaliação do Enfrentamento da Hospitalização. Li et al. ${ }^{(35)}$ realizaram uma pesquisa com crianças chinesas entre 9 a 16 anos, internadas para tratamento de câncer em unidades de oncologia pediátrica. Utilizaram entrevistas estruturadas durante um período de 8 meses, e avaliaram intervenções psicológicas eficazes que ajudaram no enfrentamento, diante do tratamento do câncer. E por último Stam at al.(37) avaliaram sobreviventes de crianças com câncer, entre 18 e 30 anos, convidados anonimamente para preencher um questionário sobre qualidade de vida, e administração da doença. Deste modo, todas as referências mencionaram técnicas e relataram sua eficácia no tratamento do câncer infantil em crianças hospitalizadas.

O objetivo do estudo foi a quarta dimensão analisada, posto que essa extensão indicou qual o objetivo dos artigos em relação à pesquisa explorada. Todas as referências objetivaram utilizar técnicas da Terapia Cognitivo-Comportamental, alternando-as.

As referências a seguir, mostraram diferentes formas de objetivos. Para Beltrão et al.(34), por exemplo, teve como objetivo a concepção materna frente ao câncer infantil. Já Morais e Enumo(32) e Motta e Enumo ${ }^{(33)}$ avaliaram os efeitos comportamentais de acordo com as intervenções lúdicas, tendo como objetivo manter o bem-estar do indivíduo através de mecanismos que amenizem os efeitos estressantes da doença e da hospitalização. Hoster et al. ${ }^{(28)}$ e Li et al. ${ }^{(35)}$ objetivaram utilizar estratégias de enfrentamento em crianças hospitalizadas com câncer e como elas conduzem a hospitalização. Motta e Enumo(29) e Motta e Enumo(30) analisaram a importância do brincar no hospital por meio de instrumentos de Avaliação do Enfrentamento da Hospitalização. Já para Hildenbrand et al. ${ }^{(36)}$ teve como objetivo explorar estressores comuns relacionados ao câncer, e como os pais se relacionam com a doença. Os mesmos consideram que o estudo do enfrentamento como elemento fundamental para compreender como o estresse afeta a vida das pessoas, principalmente o desenvolvimento 
de crianças e adolescentes. E por último Stam at al. ${ }^{(37)}$ analisaram como é a expectativa de vida de jovens e adolescentes, que já tiveram câncer na infância e explorar o papel de enfrentamento cognitivo em relação à Validação de Questionário e Avaliação da Qualidade de Vida (QVRS).

As Técnicas é a quinta dimensão de análise e podemos destacar que a maioria dos participantes dos estudos foram analisados a partir de técnicas da Terapia CognitivoComportamental, bem como, intervenções lúdicas, aplicação de instrumentos padronizados, sobretudo, por questionários e escalas.

Ressalta-se que, dessas dez referências, cada uma delas utilizou-se diferentes técnicas, bem como Beltrão et al. ${ }^{(34)}$ utilizaram técnicas de observação e entrevista gravada. O estudo de Motta e Enumo(33) empregou o Instrumento Informatizado de Avaliação do Enfrentamento da Hospitalização (AEHcomp) e o Programa de Intervenção Psicológica no Hospital (PIPH). Hoster et al. ${ }^{(28)}$ utilizaram-se de quatro métodos, o Child Behavior Checklist (618 anos), o roteiro para coleta de informações sociodemográficas dos participantes, o protocolo de consulta aos dados médicos dos participantes e o Instrumento Informatizado de Avaliação do Enfrentamento da Hospitalização. Em Motta e Enumo(29) os instrumentos utilizados foram o Instrumento de Avaliação das Estratégias de Enfrentamento da Hospitalização (AEH), as Escala de Stress Infantil - ESI, o Inventário de Ansiedade TraçoEstado - IDATE-C, a Autoquestionnaire Qualité de Vie Enfant Imagé - AUQEI de Manificat e Dazord, o Inventário de Estratégias de Copinge e a Escala de Avaliação do Comportamento da Criança. Já Motta e Enumo(31) empregou o roteiro para registro de dados familiares, o Instrumento de Avaliação de Estratégias de enfrentamento da hospitalização (AEH), a Escala de stress infantil - ESI, o Inventário de ansiedade traço-estado - IDATE-C, o Autoquestionnaire qualité de vie enfant imagé - AUQEI de manificat e dazord, o Inventário de estratégias de coping e a Escala de avaliação do comportamento da criança. Moraes e Enumo ${ }^{(32)}$ utilizou a Avaliação Informatizada do Enfrentamento da Hospitalização (AEHcomp) e o Child Behavior Checklist (CBCL 6-18 anos). Já Hildenbrand et al. ${ }^{(36)}$ prefere-se apenas a Entrevista semi-estruturada qualitativa. Li et al. ${ }^{(35)}$ utilizaram a Entrevista Estruturada. Stam et al. ${ }^{(37)}$ analisaram de quatro formas distintas, através da QVRS (Validação de questionário e avaliação da qualidade de vida), do QV (qualidade de vida), da RAND-36 (escalas) e do CCSS (Controle Escala de Estratégias cognitiva).

As duas últimas dimensões analisadas foram os resultados e as conclusões; e os dados apresentados possibilitaram apreender que a disputa de todos os artigos era identificar as técnicas e intervenções mais eficazes dentro da Terapia Cognitivo-Comportamental.

Rev. Psicol Saúde e Debate. Jan., 2017:2(2):58-75. 
Destarte, Motta e Enumo(29) concluíram que o brincar corresponde a 78,6\% das respostas relacionadas ao desejo da criança hospitalizada justificando o uso intenso da intervenção lúdica. Além disso, finalizam relatando que o instrumento se mostrou adequado à compreensão e atendimento psicológico da hospitalização. Já Hoster et al. ${ }^{(28)}$ concluiu que as crianças destacam a importância da Classe Hospitalar para a continuidade da aprendizagem e da escolaridade, considerando que ainda são insuficientes os estudos direcionados à classe. Por fim, os autores ${ }^{(28)}$ expõem a relevância de investigar as estratégias de enfrentamento utilizadas por crianças com câncer, explorando a necessidade da escola dentro do hospital.

Hoster et al. ${ }^{(28)}$ e Li et al. ${ }^{(35)}$ finalizaram através do relato das crianças, 20 estratégias de enfrentamento, com predomínio da técnica da distração. Além da distração foram identificadas estratégias de regulação emocional, quando a criança relatava uma experiência de bem-estar e/ou felicidade ao estudar. O comportamento de estudar também esteve associado à estratégia de solução do problema, quando a justificativa da criança evidenciava uma melhora de sua condição clínica ao estudar. A estratégia de reestruturação cognitiva foi registrada quando a criança se referiu ao comportamento de estudar como indicador de que aquele momento (hospitalização) era passageiro e a vida retornaria ao seu curso.

Motta e Enumo(31) finalizaram o estudo verificando que o brincar fazia parte do repertório de estratégias de enfrentamento da hospitalização da maioria das crianças (92,9\%). Já Hoster et al. ${ }^{(28)}$ ponderaram que os resultados encontrados em crianças hospitalizadas por doenças diversas, pois a hospitalização afeta o comportamento infantil e foi mediada pelas estratégias de enfrentamento de forma ímpar. Além disso, o modelo de coping mostrou comportamentos e estratégias favoráveis ao enfrentamento da hospitalização, indicando possíveis benefícios para o tratamento do câncer.

A TCC 'Terapia Cognitivo-Comportamental' oferece contribuições para o enfrentamento da hospitalização de crianças com câncer, por meio de técnicas como o brincar que se insere como uma tentativa de transformar o ambiente hospitalar, proporcionando assim melhores condições psicológicas.

Baseado nas afirmativas de Silva e Melo ${ }^{(38)}$ existe a necessidade de compreender as formas de enfrentamento, principalmente quanto o processo do adoecimento, uma vez que podem despontar dificuldades entre os familiares e o paciente. A partir disso, é essencial a intervenção junto às famílias, buscando assim, reduzir a dor e o impacto da doença. 
Sabe-se que vários métodos são utilizados para o monitoramento e diagnóstico do cancro, promovendo avanços significantes na prevenção do câncer, mas ao contatar a doença precocemente, o tratamento medicamento em conjunto com as estratégias terapêuticas da TCC torna o tratamento muito mais eficaz ${ }^{(39)}$.

\section{CONSIDERAÇÕES FINAIS}

As revisões sistemáticas possibilitam a construção de uma síntese do conhecimento científico nacional e internacional das técnicas de intervenções da Terapia CognitivoComportamental para o enfrentamento da hospitalização em crianças com câncer.

Através da criança, é possível observar o benefício do uso da brincadeira e principalmente o efeito imediato que têm, quando se alegram e se enchem de esperança durante a brincadeira. Contudo, o hospital fornece recursos para que o ambiente possa ser modificado, consequentemente, aproximando-o de sua realidade diária, derivando positivamente no processo da hospitalização. Por meio disso, as brincadeiras recreativas, livre e sem interesse, conseguem ter um resultado terapêutico interventivo, visto que amparam o processo de promoção da saúde e do bem-estar da criança.

Ao selecionar o tipo de brinquedo para cada criança no hospital, a mesmas identificam razões específicas para suas respostas, mas de um modo geral, não apresentam muitas restrições aos tipos de brincadeiras, mostrando que o importante, não é o brinquedo, e sim, a interação. Para tal, gentilezas deveriam ser aplicadas por parte das instituições, no sentido de organizar atividades recreativas que contribuem para o uso do brincar como uma estratégia de enfrentamento assertiva.

Buscando enfatizar seus esforços para enfrentar a hospitalização, entende-se que, em alguns casos, fica difícil encontrar estratégias para superar adequadamente ou positivamente os excessos aversivos a que são frequentemente expostas. A partir disso, é possível permitir que falem de sua condição, ou seja, ampliar suas oportunidades de vivenciar uma maior autonomia sobre suas próprias vidas, na proporção em que seus relatos possam conduzir mais adequadamente a intervenção.

Através dos resultados alcançados neste trabalho e possível indicar novos caminhos para estudos na área. Estudos mais dirigidos como, estratégias de enfrentamento utilizadas 
por crianças com câncer, pesquisas mais completas, que visam notificar como a criança relata enfrentar a hospitalização e como seus familiares entendem sobre o que ela faz nessas circunstâncias.

Recomenda-se a partir dessas revisões, um aprofundamento em estudos de técnicas e intervenções eficazes para o tratamento de crianças com câncer, além de brincar. Nesta perspectiva, ao finalizar a revisão, é preciso compreender que ainda há uma insuficiência de pesquisas que possam disponibilizar mais referências, em relação às crianças com câncer.

Por fim, a difusão de estudos que abordem uma revisão sistemática e os resultados de pesquisa, são elementos importantes para a construção de novos conhecimentos e desenvolvimento de instrumentos avaliados por técnicas da Terapia CognitivoComportamental. Todavia, faz-se necessária uma mudança de procedimento por parte dos pesquisadores que sugere não só o estudo intensivo, mas também a divulgação dessas informações para o exercício clínico diário. O objetivo final do processo realizado neste trabalho é desenvolver e suscitar interesse, tanto dos pesquisadores, quanto dos clínicos, em relação à existência de instrumentos essenciais que possam aprimorar e testar as técnicas de enfrentamento da hospitalização.

\section{REFERÊNCIAS}

1. Nascimento LC, Rocha SMM, Hayes VH, Lima RAG. Crianças com câncer e suas famílias. Revista da Escola de Enfermagem da USP. 2005;39(4):469-474.

2. Oliveira GF, Dantas FDC, Fonseca PN. O impacto da hospitalização em crianças de 1 a 5 anos de idade. Revista da Sociedade Brasileira de Psicologia Hospitalar. 2004;7(2):37-54.

3. Lima SSC, Botelho HRS, Silvestre MM. Câncer infantil: aspectos emocionais e o sistema imunológico como possibilidade de um dos fatores da constituição do câncer infantil. Revista da Sociedade Brasileira de Psicologia Hospitalar. 2011;14(2):142-159.

4. Souza LPS, Silva RKP, Amaral RG, Souza AAM, Mota EC, Silva CSO. Câncer infantil: sentimentos manifestados por crianças em quimioterapia durante sessões de brinquedo terapêutico. Revista da rede de enfermagem do Nordeste. 2012;13(3):686-92. 
5. Valle ERM. Câncer Infantil: Compreender e agir. São Paulo: Editorial Psy; 1997.

6. Morais RCM, Assis AC. A utilização do brinquedo terapêutico à criança portadora de neoplasia. Revista de Pesquisa: Cuidado é Fundamental Online. 2010;2(2);102-106.

7. Ferreira APQ, Lopes LQF, Melo MCB. O papel do psicólogo na equipe de cuidados paliativos junto ao paciente com câncer. Revista da Sociedade Brasileira de Psicologia Hospitalar. 2011;14(2):85-98.

8. Lipp MEN. Como enfrentar o stress infantil. São Paulo: Ícone; 1991.

9. Cohen RHP, Melo AGS. Entre o hospital e a escola: o câncer em crianças. Estilos clin. 2010;15(2):306-325.

10. Cardoso FT. Câncer infantil: aspectos emocionais e atuação do psicólogo. Revista da Sociedade Brasileira de Psicologia Hospitalar. 2007;10(1):25-52.

11. Costa JR, Áderson L. Psico-Oncologia e manejo de procedimentos invasivos em oncologia pediátrica: uma revisão de literatura. Psicologia: Reflexão e Crítica. 1999;12(1):107-118.

12. Carvalho MM. Introdução à psiconcologia. São Paulo: Psy II; 1994.

13. Cardoso FT. Câncer infantil: aspectos emocionais e atuação do psicólogo. Revista da Sociedade Brasileira de Psicologia Hospitalar. 2007;10(1):25-52.

14. Shavelzon J. Tecnicas psicoterapeuticas analíticas y no analítica en cancerología. El hospital. Apostila fornecida no II Encontro Brasileiro de Psiconcologi. Brasilia, DF; 1992.

15. Gimenes MGG. Personalidade, padrões comportamentais e câncer. Anais da XVIII Reunião Anual da Sociedade de psicologia, Ribeirão preto, 1988;173-180.

16. Beck AT, Rush AJ, Shaw BF, Gary E. Terapia cognitiva da depressão. Porto Alegre: Artmed; 1997. 
17. Falcone E. Psicoterapia cognitiva. In: B. Rangé (org) Psicoterapias cognitivocomportamentais um diálogo com a psiquiatria. Porto Alegre: Artmed; 2001.

18. Belo F, Scodeler KA. Importância do brincar em Winnicott e Schiller. Tempo psicanal. 2013;45(1):91-101.

19. Goldfried MR, Davison GC. Clinical behaviortherapy. New York: Holt. Rinehart and Winston; 1976.

20. Gosch EJ. Advancing implementation of evidence-based pratices into clinical practices: How do we get there from here. Professional Psychology: Research and Practice. 2006;37(6):606-613.

21. Miltenberger RG, Fuqua RW, Woods DW. Applying behavior analysis to clinical problems: Review and analysis of habit resersal. Journal of Applied Behavioral Analysis. North Dakota, 2006;37(3):447-469.

22. Friedberg RD, Mcclure JM. Clinical practice of cognitive therapy with children and adolescents: the nuts and bolts. New York: Guilford Press; 2002.

23. Brown CD. Therapeutic play and creative arts helping children cope with ilness, dead, and grief. Armstrong-Daily, A.; Zarboch, S. (Eds.) Hospice care for children $2^{\text {nd }}$ ed. New York: Oxford University, 2001;251-283.

24. Dell'Aglio DD. O processo de coping em crianças e adolescentes: adaptação e desenvolvimento. Temas psicol. 2003;11(1):38-45.

25. Lakatos E, Marconi M. A metodologia do trabalho científico. São Paulo: Atlas; 2010.

26. Sampaio RF, Mancini MC. Estudos de revisão sistemática: um guia para síntese criteriosa da evidência científica. Revista Brasileira de Fisioterapia. 2007;11(1):83-89.

27. Gil AC. Como elaborar projeto de pesquisa. 4⿳a ed. São Paulo: Atlas; 2002.

Rev. Psicol Saúde e Debate. Jan., 2017:2(2):58-75. 
28. Hostert P, Motta A, Enumo S, Hostert P, Motta A, Enumo S. Coping da hospitalização em crianças com câncer: a importância da classe hospitalar. Estudos de Psicologia. 2015; 32(4):627-639.

29. Motta AB, Enumo SRF. Brincar no hospital: câncer infantil e avaliação do enfrentamento da hospitalização. Psicologia, Saúde e Doenças. 2002;3(1):23-41.

30. Motta AB, Enumo SRF. Brincar no hospital: estratégia de enfrentamento da Hospitalização infantil. Estudos de Psicologia. 2004;21(3):193-202.

31. Motta $A B$, Enumo SRF. Câncer infantil: uma proposta de avaliação as estratégias de enfrentamento da hospitalização. Estudos de Psicologia. 2004;21(3):193-202.

32. Moraes EO, Enumo, SRF. Estratégias de enfrentamento da hospitalização em crianças avaliadas por instrumento informatizado. Psico-USF. 2008;13(2):221-231 .

33. Motta A, Enumo S. Intervenção psicológica lúdica para o enfrentamento da hospitalização em crianças com câncer. Psicologia: Teoria e Pesquisa. 2010;26(3):445-454.

34. Beltrão MRLR, Vasconcelos MGL, Pontes CM, Albuquerque MC. Câncer infantil: percepções maternas e estratégias de enfrentamento frente ao diagnóstico. J. Pediatria. 2007;83(6):562-566.

35. Li HC, Chung $\mathrm{OK}_{2}$ Ho $\mathrm{KY}_{2}$, Chiu $\mathrm{SY}_{2}$ Lopez $\mathrm{V}_{\text {. }}$ Coping strategies used by children hospitalized with cancer: an exploratory study. Psychooncology. 2011;20(9):969-76.

36. Hildenbrand AK, Clawson KJ, Alderfer MA, Marsac ML. Coping with pediatric cancer: strategies employed by children and their parents to manage cancer-related stressors during treatment. Psychooncology. 2011;28(6):344-54.

37. Stam $\mathrm{H}$, Grootenhuis MA, Caron $\mathrm{HN}$, Last BF. Quality of life and current coping in young adult survivors of childhood cancer: positive expectations about the further course of the disease were. Psychooncology. 2006;15(1):31-43.

38. Silva NA, Melo HCS. A Intervenção da Terapia Cognitivo-Comportamental no Adoecimento Decorrente da Insônia. Revista Psicologia e Saúde em Debate. 2015;1(1):3952. 
39. Santana NPP, Borges AR. Exames de Imagem no Rastreio e Diagnóstico do Câncer de Mama: Ressonância Magnética das Mamas em Face da Mamografia. Revista Psicologia e Saúde em Debate. 2015;1(1):20-34. 